monográfico: Trasplante Renal. Experiencia del Hospital Clínico de Barcelona

Arch. Esp. Urol., 58, 6 (503-510), 2005

\title{
VALORACIÓN DE LOS CANDIDATOS A TRASPLANTE RENAL DE DONANTE VIVO
}

\author{
Alex G utiérrez-Dalmau, Ana Saurina¹ y Anna Faura.
}

Servicio de N efrología y Trasplante Renal. Hospital Clínico de Barcelona. Servicio de N efrología ${ }^{1}$ del Hospital de Terrassa. Barcelona. España

\begin{abstract}
Resumen.- El trasplante renal de donante vivo se ha convertido en la actualidad en la opción preferencial de tratamiento sustitutivo de la función renal, siempre que sea posible su realización. En contraposición a las ventajas que ofrece en términos de supervivencia de pacientes e injertos, encontramos los riesgos asociados al hecho de la donación. En este sentido, la valoración de los candidatos a trasplante renal de donante vivo es fundamentalmente la evaluación de los riesgos ligados a la donación, para un determinado candidato, en todas las esferas, médica, psicológica, social y económica. Una evaluación de riesgos implica que tratamos un proceso conflictivo, modificable con el tiempo, el progreso médico, e incluso en el contexto familiar y/ o social de la pareja donante-receptor. La seguridad a corto y largo plazo de la nefrectomía para la donación renal va directamente ligada a la existencia de un donante sano, y este es el fin primordial de la evaluación estándar del candidato. En la actualidad y con la
\end{abstract}

Alex G uriérrez-Dalma u

Sevicio de N efrología y Trasplante Renal Hospital C linico Barcelona

C/ Villarroel, 170

08036 -Barcelona. (España)

ADALM AU@clinic.ub.es demanda creciente de esta modalidad de trasplante, la detección durante el examen de alteraciones menores o factores de riesgo no constituye siempre una contraindicación formal, pero debemos tratar de establecer a priori un umbral para la aceptación del donante 10 más objetivo posible, en las principales esferas a evaluar, tanto los riesgos quirúrgicos como aquellos relacionados directamente o no con la reducción de la masa renal, e incluso, en los donantes genéticamente relacionados, con aquellos ligados a la existencia de dicho vinculo genético entre donante y receptor, y que puede determinar el debut clínico futuro de una enfermedad renal primaria. Como en las otras modalidades de donación, el proceso de evaluación ha de asegurar también la minimización de riesgos para el receptor, con los mismos criterios de seguridad a los aplicados a los donantes cadáver. Podemos concluir que la evaluación cuidadosa del donante renal es el mejor garante para su seguridad y el éxito del trasplante, y es en nuestra opinión el mejor instrumento para ofrecer un adecuado consentimiento informado.

Palabras clave: Trasplante renal. Donante vivo. $N$ efrectomía. Evaluación de riesgo. Selección.

Summary.-Living donor kidney transplantation has become the option of preference for the treatment of endstage renal disease, whenever its performance is possible.

The advantages of patient and graft survival should be balanced with risks associated with donation. 
Therefore, the evaluation of candidates for living donor kidney transplantation is mainly the comprehensive evaluation of these risks: medical, psychological, social and economic. Evaluating risks implies we are treating a controversial process, the medical progress, which is modifiable with time, even in the family and/ or social environment of the donorreceptor couple. Short and long-term safety of living donor nephrectomy is directly engaged to the existence of a healthy donor. This he is the main objective of standard evaluation of candidates. Currently, with a growing demand of this option, minor abnormalities or risk factors detected during evaluation do not always become a formal contraindication, but we should try to establish a most objective threshold for the acceptance of donors in all evaluated spheres, for surgical risks and others directly rela ted or not with renal mass reduction, and even for those engaged to the existence of a genetic link between donor and receptor, which might determine the presence of any future primary renal disease.

As for other donation types, the process of evaluation should also ensure minimal risks for the receptor, with the same safety criteria applied to cadaver donors.

$W$ e can conclude that careful evaluation of candidates for living kidney donation is the best guarantee for their safety and transplant success, and, in our opinion, it is the best instrument to offer an adequate informed consent

Keywords: Kidney transplantation. Living donor. $N$ ephrectomy. Risk evaluation. Selection.

\section{INTRODUCCIÓN}

El trasplante renal de donante vivo posee considerables ventajas sobre el trasplante de donante cadáver. 0 btiene incrementos mayores de la supervivencia del receptor y del injerto renal $(1,2)$, a la vez que permite la realización del trasplante pre-diálisis, que suma nuevas mejoras en los dos parámetros ya mencionados $(3,4)$ y, adicionalmente, podría suponer una reducción de la carestía de órganos procedentes de cadáver disponibles para trasplante. Sin embargo, en contraposición a estos beneficios, debemos sopesar los riesgos, tanto a corto como largo plazo, asociados al hecho de la donación.

La valoración de un posible donante renal es fundamentalmente la evaluación de estos riesgos para un determinado candidato. Esta evaluación debe atender a los riesgos tanto actuales como futuros, e incluir aquellos incluso no relacionados con el proceso de donación pero que pueden determinar el futuro estado de salud del donante, como son los hábitos de vida o los factores de riesgo para el debut de diversas patologías. De este proceso derivará la decisión final de aceptación o no de dicho donante. Esta concepción de valoración del donante como una evaluación de riesgos implica el que se trate de un proceso conflictivo, modificable con el tiempo, el progreso médico, e incluso en el contexto social o personal del paciente, 0 mejor dicho, de la pareja donante-receptor. El riesgo aceptable puede diferir por ejemplo, en función del beneficio obtenido de forma indirecta por el donante con la donación, que puede ser sustancial en el caso de la donación entre esposos o mínimo o ausente en el caso de la donación entre familiares que no conviven cotidianamente con el receptor. Todo este conjunto de circunstancias establecerá finalmente un umbral de riesgo que debe guiar la toma de decisiones.

Esta evaluación debe contemplar además todas las esferas relacionadas con el posible donante, no solo la médica, si no incluyendo también aspectos sociales, económicos y sicológicos. En el presente artículo abarcamos la evaluación médica del candidato a donante renal para trasplante, mientras que los otros aspectos son tratados en otros capítulos de esta monografía. Debido a la conciencia creciente en nuestro medio de la conveniencia de esta modalidad de trasplante, cada vez es mayor la posibilidad de que se presente la donación incluso en aquellos casos en los que el examen del candidato plantee pequeñas alteraciones, por lo que hacemos especial incidencia en la delicada evaluación de estas situaciones.

\section{EVALUACIÓN ESTÁNDAR DEL DONANTE POTENCIAL}

Desde la realización del primer trasplante renal de donante vivo han transcurrido más de 50 años, que nos han demostrado que existe un riesgo mínimo, a corto y largo plazo, relacionado con la nefrectomía de un donante sano $(5,6)$. Es por tanto crucial para garantizar la seguridad del proceso la confirmación de este último término, la existencia de un donante sano, mediante la realización de un mínimo de exámenes y pruebas diagnósticas encaminadas a este propósito. 
Estas pruebas pueden ser ligeramente modificadas por las características del donante o por el contexto en que se realizan, dada la diferente disponibilidad de recursos o experiencia de los diversos centros. La Tabla I contiene una lista estándar de pruebas a las que debe ser sometido todo candidato.

Cualquier anormalidad detectada mediante estos exámenes exige una reevaluación del riesgo del donante, pudiendo derivar en pruebas adicionales, y que permitirá finalmente la toma de decisiones y la adecuada realización del consentimiento informado.

Por estas razones la selección y valoración clínica del donante debe realizarse en varias etapas, de forma ordenada, empezando con los exámenes más generales y dejando para el final las exploraciones más incómodas o sofisticadas. En ocasiones, las posibles contraindicaciones para la donación son muy evidentes (incompatibilidad de grupo sanguíneo, deter-

TABLA I. EVALUACIÓN MÉDICA ESTAN DAR DEL DO N AN TE

\author{
Historia médica completa \\ Exploración física exhaustiva \\ Tensión arterial clínica de forma repetida (3 determinaciones separadas al menos por 1 semana) y prefe- \\ rentemente, en casos seleccionados monitorización continua de tensión arterial ambula toria \\ Pruebas de laboratorio \\ Sangre: \\ Creatinina, Urea/ BUN, electrolitos, acido úrico, colesterol, triglicéridos, enzimas hepáticos, \\ biblirrubina, fosfatasa alcalina, PCR, proteinograma y equilibrio acido-base. \\ Glucemia basal y si es anormal, test de tolerancia oral a la glucosa \\ Perfil hematológico completo y pruebas de coagulación (TP y TTPA) \\ O rina: \\ Sedimento urinario, orina de 24 h con determinación de proteínas, creatinina, urea, electrolitos \\ y glucosa. \\ Inmunológicas: \\ Grupo sanguíneo, tipaje HLA, y prueba cruzada donante-receptor
}

Estimación de función renal

Aclaramiento de creatinina según orina de $24 \mathrm{~h}$ (dos ocasiones) y pruebas isotópicas en casos límite.

Estudio de enfermedades infecciosas:

Virus hepatitis B y C, HIV, HTLV, CMV, Toxoplasma, Sífilis, VEB, PPD.

Cultivo de orina

Radiología convencional de torax y abdomen

ECG. Ecocardiografía y pruebas de estrés en casos seleccionados

Pruebas de función respiratoria en fumadores y antecedentes de patología respiratoria (EPO C, asma, etc)

Anatomía renal

Ecografía de abdomen, urografía ev y angio-TAC o angio-RM

Exclusión de enfermedad tumoral (donantes mayores de 50 años) ;

PSA, exámen urológico/ ginecológico, sangre oculta en heces 
minados antecedentes patológicos, etc.) y deben esclarecerse lo antes posible para no someter al candidato a exploraciones innecesarias. Un orden lógico en las etapas de estudio del donante sería el siguiente:

\section{Primera entrevista con el donante:}

- Información general sobre la donación: riesgos, beneficios, resultados.

- Si la información está disponible, constatar que no existe incompatibilidad de grupo sanguíneo.

- Valoración de antecedentes clínicos que contraindiquen la donación (neoplasias, diabetes, hepatitis virales, etc.).

- Valoración de las características clínicas del receptor que pueden comprometer la viabilidad del injerto: riesgo de recidiva de la nefropatía original, sensibilización HLA, vasculopatía, etc.

\section{Examen inmunológico (tipaje HLA, cross-match ini-} cial):

- Muy útil para seleccionar hermanos HLA-idénticos.

- Un cross-match inicial es imprescindible si el receptor es un retrasplante o presenta sensibilización HLA.

\section{Historia clínica detallada:}

- Antecedentes de hipertensión arterial, litiasis, infecciones urinarias, proteinuria en el embarazo, cirugía abdominal, tromboembolismo venoso.

- Peso, talla, índice de masa corporal, tensión arterial.

\section{Exploraciones complementarias:}

- Análisis de sangre y orina, pruebas de imagen y demás exploraciones según se detallan en la Tabla I.

\section{Revisión urológica, valoración psicológica y visita} pre-anestésica.

\section{Cross-match definitivo.}

7. Presentación ante el Comité de Ética Asistencial.

\section{Presentación ante el Registro Civil.}

\section{RIESGO QUIRÚRGICO}

Habitualmente el riesgo quirúrgico de la donación se asimila al riesgo de la nefrectomía laparoscópica. La mortalidad de este procedimiento es baja $(0,03 \%)$ si se excluye la existencia de enfermedad cardiopulmonar significativa, no obstante hasta un $3 \%$ de los pacientes experimentan complicaciones mayores debidas a la cirugía $(7,8)$.

Como se ha mencionado, el riesgo de esta cirugía es determinado esencialmente por la situación cardiovascular del paciente, que permite establecer una serie de predictores clínicos. Recientemente, estos predictores de un riesgo incrementado en la cirugía no cardíaca han sido clasificados en diversas categorías (Tabla II) por el Colegio A mericano de Cardiología (9). En general la presencia de predictores mayores 0 intermedios contraindica la donación, mientras que los menores exigen de una consideración individualizada del riesgo y la aceptación del donante solo en casos muy seleccionados, ya que cualquier pequeño aumento del riesgo puede ser difícilmente justificado en el trasplante de donante vivo. Dada la creciente prevalencia de la enfermedad cardiovascular en nuestra sociedad y la trascendencia de su detección, parece razonable realizar pruebas adicionales al ECG (ecocardiograma y pruebas de estrés) para demostrar la ausencia de enfermedad coronaria subclínica en candidatos mayores de 40 años o con factores de riesgo cardiovascular asociados (10).

Del mismo modo, ante la existencia de factores de riesgo a sociados o una historia clínica sugerente puede ser necesaria la realización de pruebas de función pulmonar. El riesgo quirúrgico se halla incrementado ante la presencia de FEV1 < $70 \%$, FVC < $70 \%$, o un ratio FEV $1 /$ FVC $<65 \%$ (11).

TABLA II. PREDIC TO RES DE UN RIESG O Q UIRÚG IC O IN CREM EN TADO (9)

\section{Mayores}

Síndrome coronario inestable Insuficiencia cardíaca descompensada A rritmia cardíaca significativa Enfermedad valvular severa

\section{Intermedios}

Angina estable

Antecedente de infarto agudo de miocardio Insuficiencia cardíaca compensada Diabetes M ellitus

\section{Menores}

Edad avanzada ( $>60$ años)

ECG alterado o ritmo cardíaco no sinusal Baja capacidad funcional cardíaca

Antecedente de accidente cerebrovascular Hipertensión arterial no controlada 
Los individuos fumadores deben ser aconsejados con firmeza para abandonar dicho hábito, al menos 4 semanas antes de la donación, lo que disminuiría el riesgo de morbimortalidad perioperatoría (12). La misma recomendación es aplicable a los consumidores de más de 60 gramos de alcohol diario y mantenido durante un periodo superior a 6 meses (13).

\section{RIESGOS RELACIONADOS CON LA REDUC- CIÓN DE MASA RENAL}

\section{Función renal}

El grado de función renal aceptable para la donación sería aquel que asegure una función renal adecuada tanto al donante como al receptor tras el trasplante. Por tanto equivale a establecer el umbral por encima del cual, y en ausencia de enfermedad renal subyacente $u$ otros factores de riesgo, se asegura la ausencia de un deterioro acelerado de la función renal en el donante en relación a lo esperable en presencia de ambos riñones. Actualmente existe cierto consenso en considerar adecuado una tasa de filtración glomerular (TFG) mayor de $80 \mathrm{ml} / \mathrm{min}$ prenefrectomía (14).

Es obligado hacer mención a la técnica utilizada para la estimación de la TFG. Como es conocido, el cálculo del aclaramiento de creatinina basado en la recolección de orina de $24 \mathrm{~h}$ ofrece diversas posibilidades de error dependiendo de factores como la dieta, el ejercicio físico o la función renal. Por ello debe realizarse en al menos dos ocasiones, se deben valorar los resultados ajustados a la superficie corporal, y en aquellos casos en los que encontremos cifras próximas al límite de lo establecido, sería recomendable ampliar estos estudios con otras pruebas más fiables de determinación de la función renal (pruebas isotópicas, etc), e incluso de la reserva renal funcional.

\section{Diabetes Mellitus}

El diagnóstico de Diabetes Mellitus (DM) desaconseja en la actualidad la donación renal. La información disponible muestra que la nefrectomía unilateral en el paciente con DM tipo 2 con función renal normal y ausencia de proteinuria puede incrementar el riesgo de progresión de la enfermedad renal (15). Cualquier candidato con cifras dudosas de glucemia basal o factores de riesgo asociado debería ser evaluado mediante al menos un test de tolerancia oral a la glucosa.
Un punto más controvertido y de más difícil estimación, es la detección de aquellos individuos que van a desarrollar DM 2 en el futuro. La obesidad, la raza y la historia familiar son factores de riesgo relevantes, y que deben impulsar al menos a una evaluación cuidadosa del candidato en este aspecto (16). La diabetes gestacional, constituye un factor de riesgo mayor, siguiéndose del desarrollo de DM 2 hasta en un $35 \%$ de mujeres 15 años tras el parto (17), por lo que debe desaconsejar la donación en la mayoría de los casos.

\section{Hipertensión Arterial}

La HTA constituye en la actualidad una de las principales causas de IRC y necesidad de tratamiento renal sustitutivo, por lo que es de extremadamente relevante su detección previa a la donación, así como la evaluación de los posibles factores de riesgo asociados. Habitualmente se ha utilizado la TA clínica en tres determinaciones aisladas y en visitas separadas para establecer al diagnóstico de HTA. No obstante diferentes trabajos han puesto de relevancia la superioridad de la monitorización continua de la TA como método de cribaje (18). Por tanto este test debería practicarse en aquellos candidatos con valores límite en la determinación de TA clínica y en aquellos que presentan factores de riesgo asociado. Del mismo modo, sería necesario un estudio extenso de los posibles daños en órganos diana (fondo de ojo, ecocardiografía y ecografía carotidea).

Aunque la recomendación general es la no aceptación de los individuos hipertensos, es difícil establecer con precisión el riesgo de aquellos candidatos con HTA de fácil control, ausencia de microalbuminurria y función renal normal, y necesidad de un único fármaco (preferentemente un IECA con o sin tiazidas) en su tratamiento. A lgunos trabajos confirman la seguridad a corto plazo de la nefrectomía unilateral en los donantes con este perfil $(19,20)$, por lo que podría considerarse en estos individuos la donación, especialmente en aquellos con edades superiores a los 50 años.

\section{Obesidad}

La obesidad es un problema sanitario creciente, que se asocia con frecuencia a complicaciones como la diabetes y la enfermedad vascular. De este 
modo este conjunto de candidatos podría considerarse en riesgo para el desarrollo futuro de proteinuria, hipertensión, diabetes e IRC. En grandes estudios poblacionales la obesidad ha demostrado ser un factor de riesgo independiente a la HTA y la DM para el desarrollo de proteinuria (21), que como es conocido constituye uno de los principales marcadores de riesgo de desarrollo de IRC. Estudios recientes revelan incluso, que la obesidad es un factor de riesgo independiente (HTA, DM y proteinuria) para el desarrollo de IRC (22), puede acelerar la progresión de la enfermedad renal coexistente (23) y constituye un factor de riesgo para el desarrollo de proteinuria e IRC tras la realización de la nefrectomía por indicaciones diferentes a la donación (24). La obesidad constituye además un factor de riesgo independiente de morbilidad quirúrgica. De todo ello podemos concluir que es altamente recomendable la perdida de peso en pacientes obesos (BMI >30) previamente a la donación, y esta debe desaconsejarse en caso de BMI mayor a 35 y/ 0 existencia de otros factores de riesgo.

\section{Anomalías Urinarias Asintomáticas}

5.1. Proteinuria. La presencia de proteinuria significativa debe descartarse mediante la determinación de la excreción de proteínas en orina de 24 h, en al menos dos ocasiones diferentes. La presencia de una proteinuria superior a $300 \mathrm{mg} / 24 \mathrm{~h}$ supone una contraindicación para la donación renal, con la excepción de que pueda ser atribuida a proteinuria ortostática ya sea fija o persistente, definida con criterios claramente establecidos (25). Esta entidad ha demostrado un comportamiento benigno en la casi totalidad de los casos, con tendencia a la desaparición a lo largo del tiempo y mostrando estabilidad de la función renal. A un así ha de señalarse que en un bajo porcentaje de casos de proteinuria ortostática fija, se ha evidenciado la presencia de diversas nefropatías tras la biopsia $(26,27)$, por lo que ante el diagnóstico de proteinuria ortostática fija sería conveniente una evaluación meticulosa de los casos que incluya un examen histológico renal.

5.2. Microalbuminuria. Es difícil establecer recomendaciones en torno a este hallazgo de forma aislada en la evaluación del donante vivo para trasplante renal. La microalbumiruria puede ser un marcador precoz de enfermedad renal incluso en ausencia de DM2, por lo que estos candidatos merecen al menos un seguimiento más meticuloso y reiterado de lo habitual.

5.3. Microhematuria. La microhematuria puede ser un marcador de enfermedad renal subyacente como es el caso de diversas glomerulopatías. De hecho la biopsia renal en pacientes con microhematuria persistente demuestra en un elevado número de ocasiones la existencia de diversas alteraciones histológicas, y se corresponde con un examen completamente normal en menos de un $20 \%$ de las ocasiones (28). En conclusión se recomienda un examen urológico completo en estos pacientes, incluyendo la citología urinaria, seguido de la biopsia renal en caso de la ausencia de hallazgos sinificativos.

\section{Urolitiasis}

La donación renal en presencia de enfermedad litiásica debe ser evaluada con extrema precaución, puesto que la nefrectomía posiblemente constituye un factor de riesgo adicional para el desarrollo de complicaciones a largo plazo, incluyendo el deterioro funcional renal (29). Los antecedentes de litiasis recurrente, la presencia de litiasis múltiple o bilateral, la litiasis asociada a trastornos metabólicos o infección recurrente, debe contraindicar la donación. Es aceptable la presencia de un único cálculo de tamaño pequeño $(<1.5 \mathrm{~cm})$ que pueda ser fácilmente eliminado antes o durante la nefrectomía, siempre que no existan otros factores de riesgo de litiasis.

\section{Dislipemia}

Por si sola no contraindica la donación pero debe ser tenida en cuenta en la evaluación global de riesgos del donante, destacándose su papel en el incremento de la progresión de la enfermedad renal y como factor de riesgo cardiovascular.

\section{RIESGOS NO RELACIONADOS CON LA REDUCCIÓN DE MASA RENAL}

\section{Enfermedad renal primaria}

La donación entre donante-receptor genéticamente relacionados constituye una de las circunstancias todavía más frecuentes en nuestro medio, esto conlleva la posibilidad incrementada de encontrar 
patología renal, actual o futura en el donante cuando el receptor presenta una nefropatía primaria como causa de su IRC. De entre las entidades principales que nos enfrentan a este problema encontramos la poliquistosis renal autosómica dominante (PQRAD), el Síndrome de Alport, la enfermedad de la membrana basal delgada, la Nefropatia IgA, la Nefropatía Lúpica y algunas formas de GESF, aunque en la mayor parte de los casos la contribución del componente genético a estas nefropatías no ha sido claramente elucidado. En estas entidades algunos autores recomiendan la realización sistemática de biopsia renal en el donante geneticamente emparentado antes de recomendar la donación.

1.1. Síndrome de Alport En este caso es necesaria la exclusión exhaustiva y reiterada de proteinuria o hematuria. El diagnóstico de exclusión requiere la biopsia renal y el examen mediante microscopia electrónica

\subsection{Enfermedad de la membrana basal del-}

gada. Diversas investigaciones han puesto de relieve un curso no benigno de esta entidad, con incremento del riesgo de enfermedad renal progresiva en estos pacientes (30). Su presencia en el estudio de posibles candidatos debe por lo general excluir la donación, y tan solo aquellos casos con un menor riesgo podrían ser considerados; edad mayor de 50 años y función renal normal. La sospecha de que esta entidad es la causante de la IRC del posible receptor es un marcador de una evolución no benigna en sus familiares.

1.3. Poliquistosis renal (PQRAD). A ctualmente contamos con métodos diagnósticos de elevada fiabilidad para descartar la presencia de esta entidad en el donante, concretamente la sensibilidad de la ecografía es del $100 \%$ para su detección en individuos de riesgo y mayores de 30 años de edad (31). En candidatos más jóvenes debe en lo posible buscarse otros posibles donantes, y en todo caso puede recurrirse a técnicas más sensibles para la detección de quistes renales (TAC y RM), siempre teniendo en cuenta que no se han establecido criterios claros para la exclusión de la enfermedad en personas jóvenes.

\section{Hepatitis víricas}

Los donantes con serología positiva para el virus de la hepatitis $C$ deben evaluarse cuidadosamen- te, por el riesgo de desarrollar hepatopatía crónica 0 transmisión de la infección. Si el receptor fuese también VHC positivo, con PCR positiva, podría considerarse la donación siempre y cuando el donante tuviera una biología hepática estrictamente normal, la PCR fuera negativa y no existiera evidencia de hepatitis crónica o cirrosis.

Los donantes con antígeno B de superficie positivo deben ser generalmente excluidos. Si el antígeno es negativo pero aparecen anticuerpos contra el core, deberá cuantificarse el DNA del virus por PCR. Si éste fuera negativo podría aceptarse la donación, previa confirmación de la correcta inmunización del receptor frente al antígeno de superficie.

\section{Neoplasias}

La exclusión de la enfermedad neoplásica debe ser una prioridad en el donante mayor de 50 años. Los criterios de aceptación de un donante con antecedentes de neoplasia maligna podrían ser similares a los empleados en la aceptación del donante cadáver, siempre que el tratamiento del cáncer no suponga un incremento del riesgo quirúrgico o renal tras la nefrectomía. Los antecedentes de neoplasia constituyen de forma global una contraindicación para la donación, salvo si se trata de tumores cutáneos de tipo no melanoma y bajo grado, carcinoma de cervix in situ, o si la transmisión del cáncer puede ser razonablemente excluida como es el caso del cáncer de colon de bajo grado hace más de 5 años.

\section{CONCLUSIONES}

Como resumen debemos recalcar la extremada importancia de la evaluación cuidadosa del donante renal para su seguridad y el éxito del trasplante, y es en nuestra opinión el mejor instrumento para minimizar los riesgos que afronta el donante y ofrecerle un adecuado consentimiento informado.

Fijar un umbral de riesgo aceptable es enormemente difícil para todos los casos, y aunque es obligada la potenciación de esta modalidad de donación en nuestro medio, ello no debe significar el incremento de este umbral de forma indiscriminada. 


\section{BIBUOGRAFIA y LECTURAS RECOMENDADAS (*lectura de interés y $* *$ lectura fundamental)}

*1. HARIHARAN, S.; JOHNSON, C.P.; BRESNAHAN, B.A. y cols.: "Improved graft survival after renal transplantation in the United States, 1988 to 1996". N. Engl. J. Med., 342: 605, 2000.

2. MEDIN, C.; ELINDER, C.G.; HYLANDER, B. y cols.: "Survival of patients who have been on a waiting list for renal transplantation". Nephrol Dial Transplant, 15: 701, 2000.

3. PAPALOIS, V.E.; MOSS, A.; GILLINGHAM, K.J. y cols.: "Pre-emptive transplants for patients with renal failure: an argument against waiting until dialysis". Transplantation, 70: 625, 2000.

*4. MANGE, K.C.; JOFFE, M.M.; FELDMAN, H.I.: "Effect of the use or nonuse of long-term dialysis on the subsequent survival of renal transplants from living donors". N. Engl. J. Med., 8: 726, 2001.

**5. NAJARIAN, J.S.; CHAVERS, B.M.; MCHUGH, L.E.: "A 20 years or more of follow-up of living kidney donors". Lancet, 3: 807, 1992.

**6. KASISKE, B.L.; MA, J.Z.; LOUIS, T.A. y cols.: "Long-term effects of reduced renal mass in humans". Kidney Int., 48: 814, 1995.

**7. ELLISON, M.D.; MCBRIDE, M.A.; EDWARDS, E.B. y cols.: "Living organ donation: Mortality and earlycomplications among 16,395 living donors in the US”. Am. J. Transplant, 2: 2a, 2002.

**8. MATAS, A.J.; BARTLETT, S.T.; LEICHTMAN, A.B.: "Morbidity and mortality after living kidney donation, 1999-2001: survey of United States transplant centers". Am. J. Transplant, 3: 830, 2003.

9. KIBERD, B.A.; CLASE, C.M.: "Cumulative risk for developing end-stage renal disease in the US population”. J. Am. Soc. Nephrol., 13: 1635, 2002.

10. HARTMANN, A.; FAUCHALD, P.; WESTLIE, L.: "The risk of living kidney donation". Nephrol Dial Transplant, 18: 871, 2003.

11. SMETANA, G.W.: "Preoperative pulmonary evaluation”. N. Engl. J. Med., 340: 937, 1999.

12. PEARCE, A.C.; JONES, R.M.: "Smoking and anesthesia: preoperative abstinence and perioperative morbidity". Anesthesiology, 61: 576, 1984.

13. TONNESEN, H.; ROSENBERG, J.; NIELSEN, H.J.: "Effect of preoperative abstinence on poor postoperative outcome in alcohol misusers: randomised controlled trial". BMJ., 15; 318: 1311, 1999.

**14. DAVIS, C.L.: "Evaluation of the living kidney donor: current perspectives". Am. J. Kidney Dis., 43: 508, 2004.

*15. SILVERIO, S.P.; DA COSTA, L.A.; BECK, M.O. y cols.: "Urinary albumin excretion rate and glomerular filtration rate in single-kidney type 2 diabetic patients". Diabetes Care, 121: 1521, 1998.

16. GADSBY, R.: "Epidemiology of diabetes". Adv. Drug Deliv. Rev., 54: 1165, 2002.
17. LINNE, Y.; BARKELING, B.; ROSSNER, S.: "Natural course of gestational diabetes mellitus: long term follow-up of women in the SPAWN study". BJOG., 109: 1227, 2002.

18. ROAKE, J.A.; CAHILL, A.P.; GRAY, C.M. y cols.: "Preemptive cadaveric renal transplantation-clinical outcome". Transplantation, 62: 1411, 1996.

*19. TEXTOR, S.C.; TALER, S.J.; LARSON, T.S. y cols.: "Blood pressure evaluation among older living kidney donors". J. Am. Soc. Nephrol., 14: 2159, 2003.

20. TEXTOR, S.C.; TALER, S.J.; PRIETO, M. y cols.: "Hypertensive living renal donors have lower blood pressures and purinary microalbumin one year after nefrectomy". Am. J. Transplant, 3: 192 2003.

21. TOZAWA, M.; ISEKI, K.; ISEKI, C.: "Influence of smoking and obesity on the development of proteinuria”. Kidney Int., 62: 956, 2002.

22. ISEKI, K.; IKEMIYA, Y.; KINJO, K. y cols.: "Body mass index and the risk of development of end-stage renal disease in a screened cohort". Kidney Int., 65: 1870, 2004.

23. PRAGA, M.; HERNANDEZ, E.; MORALES, E. y cols.: "Clinical features and long-term outcome of obesity-associated focal segmental glomerulosclerosis". Nephrol Dial Transplant, 16: 1790, 2001.

*24. PRAGA, M.; HERNANDEZ ,E.; HERRERO, J.C. y cols.: "Influence of obesity on the appearance of proteinuria and renal insufficiency after unilateral nephrectomy”. Kidney Int., 58: 2111, 2000.

*25. SPRINGBERG, P.D.; GARRETT, L.E. Jr.; THOMPSON, A.L. Jr. y cols.: "Fixed and reproducible orthostatic proteinuria: results of a 20-year follow-up study". Ann Intern. Med., 97: 516, 1982.

26. ROBINSON, R.R.; GLOVER, S.N.; PHILLIPPI, P.J. y cols.: "Fixed and reproducible orthostatic proteinuria. I. Light microscopic studies of the kidney". Am. J. Pathol., 139: 291, 1961.

27. ROBINSON, R.R.; ASHWORTH, C.T.; GLOVER, S.N. y cols.: "Fixed and reproducible orthostatic proteinuria. II. Electron microscopy of renal biopsy specimens from five cases". Am. J. Pathol., 39: 405, 1961.

*28. HALL, C.L.; BRADLEY, R.; KERR, A. y cols.: "Clinical value of renal biopsy in patients with asymptomatic microscopic hematuria with and without low-grade proteinuria”. Clin. Nephrol., 262: 267,2004

29. LEE, Y.H.; HUANG, W.C.; CHANG, L.S, y cols.: "The long-term stone recurrence rate and renal function change in unilateral nephrectomy urolithiasis patients". J. Urol., 152: 1386, 1994.

*30. NIEUWHOF, C.M.; DE HEER, F.; DE LEEUW, P. y cols.: "Thin GBM nephropathy: premature glomerular obsolescence is associated with hypertension and late onset renal failure". Kidney Int., 51: 1596, 1997.

*31. NICOLAU, C.; TORRA, R.; BADENAS, C. y cols.: "Autosomal dominant polycystic kidney disease types 1 and 2: assessment of US sensitivity for diagnosis". Radiology, 213: 273, 1999. 